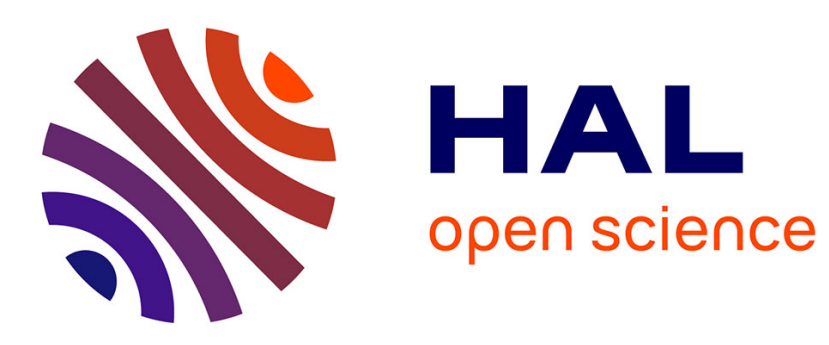

\title{
Multiscale proper generalized decomposition based on the partition of unity
}

Rubén Ibáñez Pinillo, Amine Ammar, Elías G. Cueto, Antonio Huerta, Jean Louis Duval, Francisco Chinesta

\section{To cite this version:}

Rubén Ibáñez Pinillo, Amine Ammar, Elías G. Cueto, Antonio Huerta, Jean Louis Duval, et al.. Multiscale proper generalized decomposition based on the partition of unity. International Journal for Numerical Methods in Engineering, 2019, 120 (6), pp.727-747. 10.1002/nme.6154 . hal-02545484

\section{HAL Id: hal-02545484 \\ https://hal.science/hal-02545484}

Submitted on 17 Apr 2020

HAL is a multi-disciplinary open access archive for the deposit and dissemination of scientific research documents, whether they are published or not. The documents may come from teaching and research institutions in France or abroad, or from public or private research centers.
L'archive ouverte pluridisciplinaire HAL, est destinée au dépôt et à la diffusion de documents scientifiques de niveau recherche, publiés ou non, émanant des établissements d'enseignement et de recherche français ou étrangers, des laboratoires publics ou privés. 


\title{
Multiscale proper generalized decomposition based on the partition of unity
}

\author{
Rubén Ibáñez ${ }^{1,4,6} \mid$ Amine Ammar $^{2}$ | Elías Cueto ${ }^{3}$ (D) | Antonio Huerta ${ }^{4}$ | \\ Jean-Louis Duval ${ }^{5}$ | Francisco Chinesta ${ }^{1}$
}

${ }^{1}$ ESI Chair, Laboratoire PIMM, Arts et Metiers, CNRS, CNAM, HESAM

Universite, Paris, France

${ }^{2}$ ESI Chair, LAMPA, Arts et Metiers, Angers, France

${ }^{3}$ Aragon Institute of Engineering Research, Universidad de Zaragoza, Zaragoza, Spain

${ }^{4}$ Laboratori de Calcul Numeric, Universitat Politecnica de Catalunya, BarcelonaTech, Barcelona, Spain

${ }^{5}$ ESI Group, Rungis, France

${ }^{6}$ ICI Institute, ECN, Nantes, France

\section{Correspondence}

Francisco Chinesta, Laboratoire PIMM, Arts et Metiers, 151 Boulevard de l'Hopital, 75013 Paris, France.

Email: Francisco.Chinesta@ensam.eu

\section{Funding information}

European Union's Horizon 2020 research and innovation program; Marie

Sklodowska-Curie, Grant/Award Number: 675919; Spanish Ministry of Economy and Competitiveness, Grant/Award Number: DPI2015-72365-EXP; Regional

Government of Aragon and the European Social Fund (research group T24 17R)

\begin{abstract}
Summary
Solutions of partial differential equations could exhibit a multiscale behavior. Standard discretization techniques are constraints to mesh up to the finest scale to predict accurately the response of the system. The proposed methodology is based on the standard proper generalized decomposition rationale; thus, the PDE is transformed into a nonlinear system that iterates between microscale and macroscale states, where the time coordinate could be viewed as a 2D time, representing the microtime and macrotime scales. The macroscale effects are taken into account because of an FEM-based macrodiscretization, whereas the microscale effects are handled with unidimensional parent spaces that are replicated throughout the domain. The proposed methodology can be seen as an alternative route to circumvent prohibitive meshes arising from the necessity of capturing fine-scale behaviors.
\end{abstract}

\section{KEYWORDS}

partition of unity, proper generalized decomposition, time multiscale

\section{1 | INTRODUCTION}

Many problems in computational mechanics present a multiscale behavior where the microscale effects influence the macroscale ones and vice versa. Hence, the treatment of the different scales of the problem becomes very important to reach an accurate solution. Plenty of effort has been dedicated to deal with small-scale effects either refining the mesh or introducing models that accounts for the subgrid effects. As a typical example, different levels of turbulence models can be found in, eg, Reynolds averaged Navier-Stokes, large-eddy simulation, or direct Navier-Stokes. ${ }^{1}$ In the solid domain, such a multiscale behavior appears, for instance, in the spatial domain when dealing with metamaterials whose structure is defined at the microlevel. 
Several techniques have been applied throughout history, such as the classic homogenization, ${ }^{2}$ which defines the microscale constitutive model in terms of a representative volume that satisfies the Hill-Mandel principle. ${ }^{3}$ This methodology has proven to be very effective to circumvent the prohibitive computational cost of methods like $F E^{2}$. However, there is a strong hypothesis behind such homogenization technique, requiring a clear separation of scales between macro and micro effects.

Another way to handle these multiscale effects is by using the variational multiscale framework introduced by Hughes et al, ${ }^{4}$ where the effect of the microscale into the macroscale variables is introduced in a consistent way developing different weak forms associated with the macroscale and microscale. This approach has been effectively applied in many problems such as the Navier-Stokes equation, ${ }^{5}$ among others.

Moreover, the multiscale problem will appear when dealing with temporal evolutions that are composed by many harmonics at very different frequencies. Following standard time-marching approaches, a suitable time step that captures the evolution of the finest scale has to be imposed, deriving into a prohibitive simulation cost. Many numerical algorithms have been proposed to dribble the time-marching approach up to the finest time scale.

Passieux et $\mathrm{al}^{6}$ proposed a numerical algorithm based on the LATIN-PGD, able to handle temporal and spatial multiscale behaviors appearing in solid mechanic problems. However, selecting the temporal macrobasis just like the interfacial degrees of freedom (dofs) coupling different macrodomains is very problem dependent. The scalability of the methodology is restored via an appropriate correction of the temporal basis based on the residual.

Fritzen et al ${ }^{7,8}$ also partitioned the time domain into subintervals, where a common reduced basis is applied for all subintervals. Afterwards, additional interface restrictions have to be imposed to ensure continuity of the primal variable and the time derivative. Hence, variables at both extremes of each macrointerval are set to zero, plus an offset calculated from the previous macrointerval. Several viscoelastic problems are solved for various cyclic loading with varying frequency.

Maday et $\mathrm{al}^{9,10}$ developed a Parareal algorithm that also attempts to solve a temporal evolution iterating between macrodomain and microdomain partitions so that the initial conditions for each microinterval are given by the macroresolution of the problem. The algorithm is also highly parallelizable, making it very efficient for either long time simulations or temporal problems involving many spatial dofs.

Ammar et $\mathrm{al}^{11}$ proposed an efficient technique based on the separation of variables, where the time domain was partitioned into discrete subdomains following the standard proper generalized decomposition (PGD) rationale, ${ }^{12-15}$ or equivalent reduced order approaches. ${ }^{16-18}$ However, special attention had to be paid at the interfaces between discrete subdomains, where continuity was imposed by means of Lagrange multipliers.

Badias et $\mathrm{a}^{19}$ developed an algorithm where the time domain was partitioned into different PGDs, but again, the interface conditions between different subdomains could become a cumbersome task. Another numerical strategy based on combining different PGDs under the partition of unity (PU) rationale ${ }^{20,21}$ was developed in the work of Ibáñez et al. ${ }^{22}$ The idea of using macro shape functions that respect the PU allowed the method to combine different PGDs in a consistent manner. Moreover, the overlap between PGDs automatically solves the problem of interface compatibilities related to the not overlapped methodology. However, there may be situations in which the macrodomain partition requires many PGDs to capture properly the behavior, reducing the scalability of the method.

The methodology proposed in this work aims to treat multiscale problems by means of the separation of variables paradigm, where no need to impose a clear separation between scales is required. Indeed, the methodology is based on two main pillars: a generalization of the idea of time-domain partition and the overlapped multi-PGD framework. By doing that, the proposed methodology naturally inherits continuity between macro subdomains, clear extrapolation to higher-dimensional spaces and scalability of the method even if the macrodomain partition is fine enough.

The paper is structured into four distinct sections. After this introduction, we introduce the theoretical description of the just-developed method. In Section 3, we introduce a long list of numerical examples that show the potentialities of the proposed method. Finally, in Section 4, we draw some conclusions and explore the future research lines. Details of the implementation are given in the Appendix.

\section{2 | BASICS OF THE METHOD}

This section explains the main characteristics of the multiscale PGD based on the PU. The formulation is introduced for a one-dimensional variable first. Then, the formulation is extended to $2 \mathrm{D}$ problems, and finally, to time-multiscale problems. 
Let us assume that a given function, $u(x)$, is the solution of a given partial differential equation. In terms of standard approximation basis, such as finite elements, it could be expressed as

$$
u(x)=\sum_{i=1}^{N} N_{i}(x) u_{i}
$$

where $N$ stands for the number of dofs used in the approximation of $u(x)$ or, in other words, the number of nodes in the mesh. Needless to say, $N_{i}(x)$ represents the standard finite element shape functions and $u_{i}$ represents the nodal value of the sought function, if the employed approximation is interpolant.

However, if the solution presents a multiscale approach, the mesh has to capture the details of the solution at the finest scale, thus deriving into a prohibitive simulation $\operatorname{cost}(N \gg 1)$. A possible way to circumvent this issue, within the PU paradigm, is to introduce a dependent variable that captures the solution details associated with the finest scale,

$$
u(x)=\sum_{i=1}^{N} N_{i}(x) u_{i} \sum_{j=1}^{J} G_{j}\left(\tau\left(x-x_{i}\right)\right) g_{j},
$$

where $x_{i}$ is the centroid of the shape function $N_{i}(x), \tau\left(x-x_{i}\right)$ is a dependent variable that presents an offset based on $x_{i}, G_{j}(\tau)$ is the $j$ th microscale shape function, and $g_{j}$ is its associated microscale degree of freedom. Indeed, the microscale effects occurring in the compact support of the macro shape function $N_{i}(x)$ are going to be mapped into the same microscale parent space, $\tau$.

There are several ways to define the parent space. In the particular case where all macro shape function supports are identical, the easiest choice is to equal the length of the parent space with the length associated with the macroscale shape function support, requiring only an offset based on the centroid of the $i$ th macroscale shape function. If the macro partition is not uniform, each macroscale shape function support has to be mapped to the same reference parent space. Thus, the Jacobian of this mapping will appear in the definition of the $\tau$ variable. This particular case is not considered in the present work, which looks for the simplest and more general case, even if as considered later, it is not the most efficient. Future works will address adaptivity and consequently will consider this issue.

Remark 1. It is worth noting that, in Equation (2), macro dofs $\left(u_{i}\right)$ and micro dofs (defining the local microenrichment, $g_{j}$ ) are both unknown, in opposition to usual applications of PU, where the enrichment functions are known and given a priori.

Figure 1 shows the shape functions associated with both the macroscale (top) and the microscale (bottom). Notice how a two-scale approach presents two meshes related to microscales and macroscales, respectively. Indeed, each macro partition of the domain contributes to the parent microscale, and the parent micro space affects equally the macro partition. It is important to reckon that both the macromesh and the micromesh are defined by the user. To improve the efficiency of the methodology, they should meet certain requirements as the ones listed below.

- The microscale mesh should be defined in such a way that it captures the finest microscale behavior. For instance, if the signal has a given maximum frequency, the microscale mesh has to be defined in a way that this maximum frequency is well represented, according to the Niquist-Shanon sampling theorem. It is important also to highlight that refining the microstate mesh also could increase the number of fixed-point iterations between macroscale and microscale states, as the nonlinear system becomes bigger.

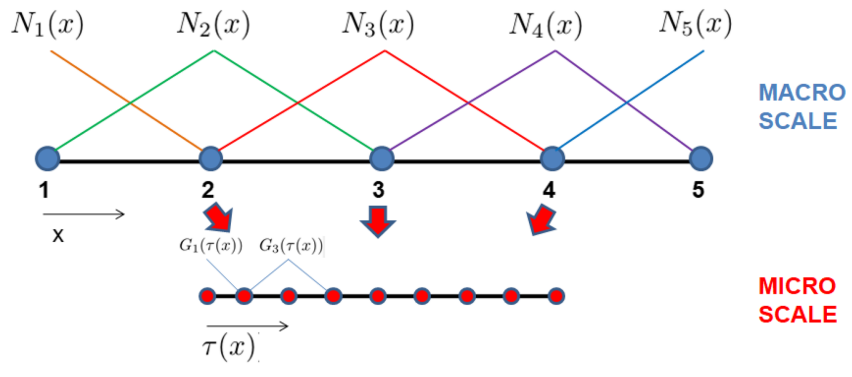

FIGURE 1 Shape functions of a multiscale approach. (Top) macro shape functions. (Bottom) micro shape functions [Colour figure can be viewed at wileyonlinelibrary.com] 
FIGURE 2 A separation of dependent variables, $u(x, \tau(x))$ [Colour figure can be viewed at wileyonlinelibrary.com]

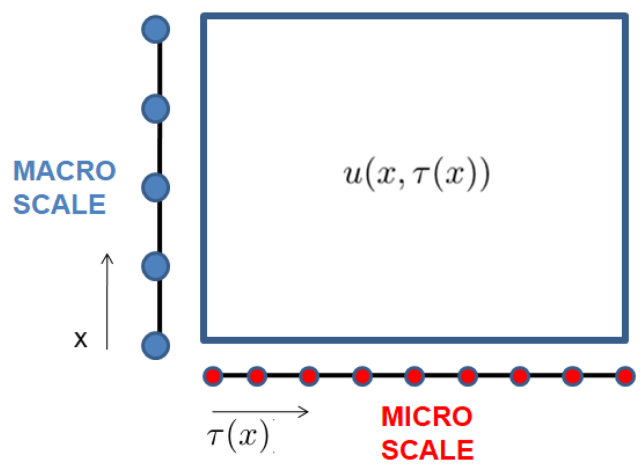

- The more a macromesh is partitioned, the simpler the microscale modes are because the signal variation is captured by either the macromesh or the micromesh. Indeed, the more partitioned a macro mesh is, the more variation could be described only with the macro elements, letting less variation to be captured by the microscale mesh.

Moreover, it is worth mentioning the possibility of rewriting our solution function as $u(x, \tau)$, making it suitable for a PGD-like algorithm. ${ }^{23,24}$ In this case, we proceed with a separation of variables, as shown in Figure 2. Therefore, the initial one-dimensional problem is transformed into a two-dimensional problem. It is important to highlight that the macromesh does not have to capture the solution of the finest scale because it is handled by the microscale mesh.

Thus, following a standard PGD rationale, ${ }^{25,26}$ the solution is sought in a Greedy manner through of a finite sum of $M$ modal enrichments,

$$
u(x, \tau)=\sum_{m=1}^{M} \sum_{i=1}^{N} N_{i}(x) u_{i}^{m} \sum_{j=1}^{J} G_{j}\left(\tau\left(x-x_{i}\right)\right) g_{j}^{m}=\sum_{m=1}^{M} \sum_{i=1}^{N} N_{i}(x) u_{i}^{m} \mathbf{G}^{T}\left(\tau\left(x-x_{i}\right)\right) \mathbf{g}^{m}=\sum_{m=1}^{M} u^{m}(x, \tau),
$$

where the super-index $m$ indicates the $m$ th PGD mode.

The discretization of a variational formulation by means of FEM, for instance, also needs an approximating space for the admissible variation of the field. In this particular case, when computing the $M$ th mode, we used a standard Galerkin projection

$$
w^{*}(x, \tau)=\sum_{i=1}^{N} N_{i}(x) u_{i}^{*} \mathbf{G}^{T}\left(\tau\left(x-x_{i}\right)\right) \mathbf{g}^{M}+\sum_{i=1}^{N} N_{i}(x) u_{i}^{M} \mathbf{G}^{T}\left(\tau\left(x-x_{i}\right)\right) \mathbf{g}^{*} .
$$

Nevertheless, streamline-upwind Petrov-Galerkin (SUPG) or Galerkin least-squares stabilization can also be implemented in this formulation when dealing with highly convective (parabolic, in general) problems. ${ }^{27}$

Even if any linearization technique is possible, an alternated direction scheme is chosen in this work for solving the resulting nonlinear problem arising from the PGD rationale. Hence, when solving microscale modes, macroscale admissible variations are set to zero (ie, $u_{i}^{*}=0$ ) and, conversely, when solving macroscale modes, $\mathbf{g}^{*}=\mathbf{0}$.

Special attention needs to be paid when dealing with the derivatives of the approximation space, viz,

$$
\frac{D u(x, \tau)}{D x}=\frac{\partial u(x, \tau)}{\partial x}+\frac{\partial u(x, \tau)}{\partial \tau} \frac{\partial \tau}{\partial x}=\sum_{m=1}^{M} \sum_{i=1}^{N}\left(\frac{\partial N_{i}(x)}{\partial x} u_{i}^{m} \mathbf{G}^{T}(\tau) \mathbf{g}^{m}+N_{i}(x) u_{i}^{m} \frac{\partial \mathbf{G}^{T}(\tau)}{\partial \tau} \frac{\partial \tau}{\partial x} \mathbf{g}^{m}\right)
$$

shows the application of the chain rule to account for the dependance of $\tau\left(x-x_{i}\right)$. For the sake of simplicity, the dependance of $\tau$ with respect to $x$ coordinate will be set to one, as we are analyzing the case in which the parent space length coincides with each one of the macroscale shape function support length; otherwise, the Jacobian of the transformation should be used.

The same rationale can be easily applied to higher-dimensional spaces. Imagine that a 2D case (ie, $\mathbf{x}=(x, y))$ is approximated by means of a 2D macromesh, and the details of the microscale are given by one-dimensional modes acting along each spatial direction. Hence, the approximation space will read

$$
u\left(\mathbf{x}, \tau_{x}, \tau_{y}\right)=\sum_{m=1}^{M} \sum_{i=1}^{N} N_{i}(\mathbf{x}) u_{i}^{m} \mathbf{G}^{T}\left(\tau_{x}\right) \mathbf{g}^{m} \mathbf{H}^{T}\left(\tau_{y}\right) \mathbf{h}^{m},
$$




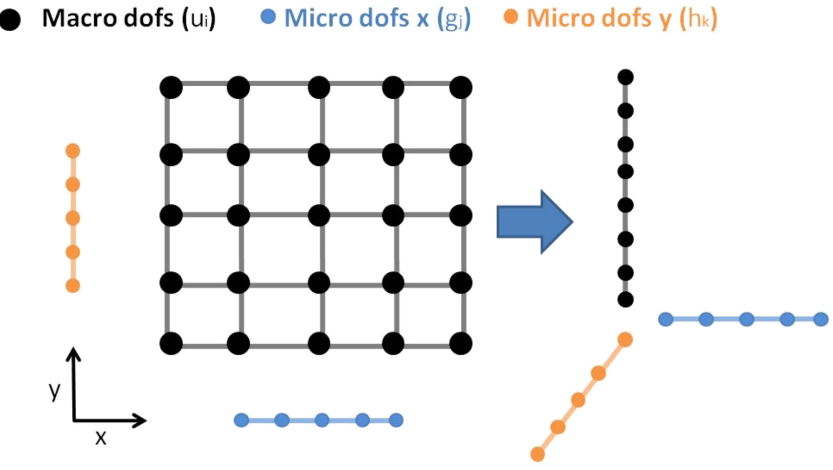

FIGURE 3 A separation of dependent variables in a 2D space, $u\left(\mathbf{x}, \tau_{x}, \tau_{y}\right)$. dofs, degrees of freedom

where $\mathbf{g}^{m}$ and $\mathbf{h}^{m}$ are the dofs of the $m$ th PGD mode acting on $x$ and $y$ subscales, respectively. Indeed, $u_{i}$ adopts the same role than in the $1 \mathrm{D}$ case because it will be responsible of weighting the subgrid behavior throughout the domain.

The separated representation by means of PU-based PGD in higher dimensions is depicted in Figure 3. Note that an initial 2D problem is transformed into a 3D problem, where one dimension takes into account the macrovariations of the solution, whereas the other two dimensions take into account the subgrid scales taking place along the $x$-and $y$-directions, respectively. It is very important to notice that subgrid scales are going to propagate throughout the entire domain, with the macromesh being responsible of controlling the propagation of the subgrid behavior.

It is also important to highlight that the proposed approach reduces the amount of dofs required to handle subgrid scales in comparison with standard multigrid methods. ${ }^{28}$ Imagine that a 2D domain is partitioned using a coarse regular mesh composed of $D_{x} \times D_{y}$ linear elements. Let us assume that each element is refined even more to handle subgrid scales using a mesh of $d_{x} \times d_{y}$ linear elements. The typical size of the entire system will be $D_{x} \times D_{y} \times d_{x} \times d_{y}$. Nevertheless, the proposed methodology is able to handle subgrid scales at a computer cost proportional to $M\left(\left(D_{x} \times D_{y}\right)+d_{x}+d_{y}\right)$. Indeed, if the number of modes required to represent the solution does not grow too much (ie, $M=\mathcal{O}(10)$ ), computing subgrid scales becomes affordable.

\section{3 | NUMERICAL EXAMPLES}

In this section, several numerical examples based on the multiscale PGD formulation are tested. The first part shows the convergence of the method for three different one-dimensional cases. The second part shows several examples of the proposed methodology for different two-dimensional cases.

Following standard procedures in the PGD rationale, two stopping criteria are required. The first one stops the alternated direction (or fixed point) scheme within the $m$ th mode computation step and is required to solve the nonlinearity arising from the separation of variables. The second stopping criteria is required to stop computing new modes. In this analysis, we have defined these two stopping criteria as

$$
\mathcal{E}_{f p}^{m, i}=\sqrt{\frac{\int_{\Omega}\left(u^{m, i}-u^{m, i-1}\right)^{2} d \Omega}{\int_{\Omega}\left(u^{m, i}\right)^{2} d \Omega}}<\operatorname{tol}_{f p},
$$

and

$$
\mathcal{E}_{\text {mode }}^{m}=\sqrt{\frac{\int_{\Omega}\left(u^{m}\right)^{2} d \Omega}{\int_{\Omega}\left(u^{1}\right)^{2} d \Omega}}<\text { tol }_{\text {mode }},
$$

where superindex $m$ stands for the $m$ th mode and superindex $i$ is related to the $i$ th fixed-point iteration.

\subsection{One-dimensional numerical examples}

In this first section, we analyze three different toy problems in one dimension. These include approximation, diffusion, and convection problems. 


\subsection{1 | Multiscale approximation of a given function}

This first example concerns the approximation of a function that shows multiscale features. Since we deal with an approximation case, there is no governing partial differential equation in this problem. In other words, a given function $f(x)$ is to be approximated by means of a multiscale approximation $u(x, \tau)$. The weighted residual form associated with this problem reads

$$
\int_{\Omega} w^{*}(x, \tau) u(x, \tau) d x=\int_{\Omega} w^{*}(x, \tau) f(x) d x \quad \forall x \in \Omega=[0,1] .
$$

In this case, the function to approximate is defined as

$$
f(x)=\frac{x \cos (8 \pi x)}{8 \pi} .
$$

Figure 4 shows the reconstructed solution (red line) versus the reference solution (blue line) when the macrodomain is partitioned using five dofs (black dots), ie, $N=5$. The microdomain is partitioned using 80 linear finite elements, ie, $J=81$. As it can be noticed, the macro partition coincides exactly with the period of the signal, which is precisely the reason why only one mode $(M=1)$ is required to accurately represent the solution. Indeed, the microscale mode is giving us the cosine signal, whereas the macroscale is taking care of the linear growth.

Figure 5 shows the relative error in logarithmic scale between the reconstructed solution and the reference solution as a function of PGD modes for the 1D approximation case with macrodomain partition coincident with the signal period. Note how, with only one mode, the multiscale PGD presents a low relative error. The same can be appreciated if the weight of the different PGD modes are compared; the first mode is almost four orders of magnitude bigger than the other two.

Figure 6 shows the convergence of the alternated direction algorithm for each one of the modes in the 1D approximation case with macrodomain coincident with the signal period. As it can be seen, all modes present a fast convergence till the stopping criterion $1 e-3$, with the first mode being the fastest one.

However, the exact period of our signal is not known a priori in most cases. Therefore, convergence of the method when the macro partition does not coincide with the signal period is checked as well. Figure 7 shows the reconstructed solution (red line) against the reference one (blue line) when the macrodomain partition does not coincide with the signal period. The top figure, involves a reconstructed solution with one mode, whereas the bottom one involves five modes. It is important to notice that the one-mode solution captures the main trend of the signal. However, there are some regions where the signal is not properly captured. Indeed, the solution involving five modes reproduces the reference signal very accurately.

Figure 8 shows the relative error in logarithmic scale with respect to the reference solution as a function of the PGD modes for the 1D approximation case where the macrodomain is not coincident with the signal period. The method converges monotonically toward the reference solution. The same decay can be appreciated comparing the weight of different PGD modes.

FIGURE 4 Approximation problem. The reconstructed solution with one single mode is represented by the red line. The reference solution is the blue line. In this case, the macrodomain partition employed five degrees of freedom, represented as black dots

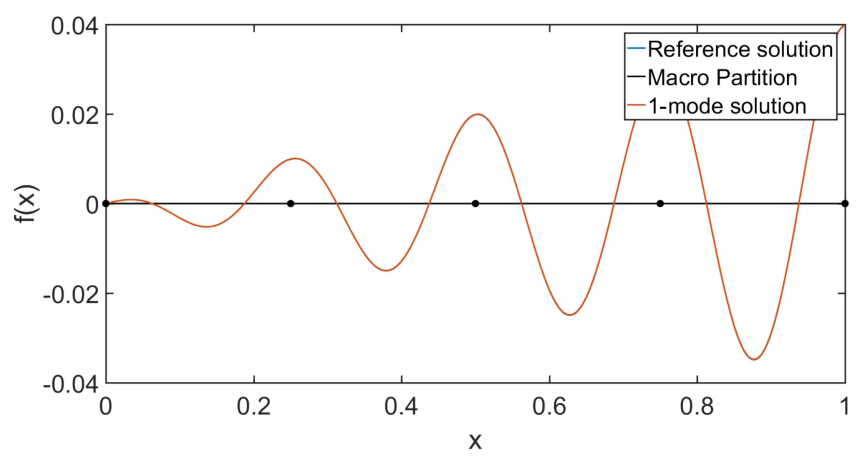

FIGURE 5 One-dimensional approximation problem macro partition coincident with a signal period. (Left) relative error with respect to the reference solution as a function of the number of proper generalized decomposition (PGD) modes. (Right) weight of each computed PGD mode [Colour figure can be viewed at wileyonlinelibrary.com]
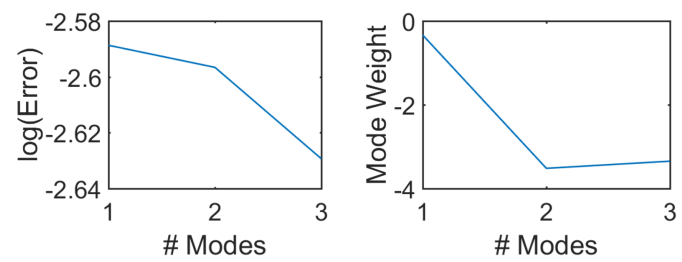

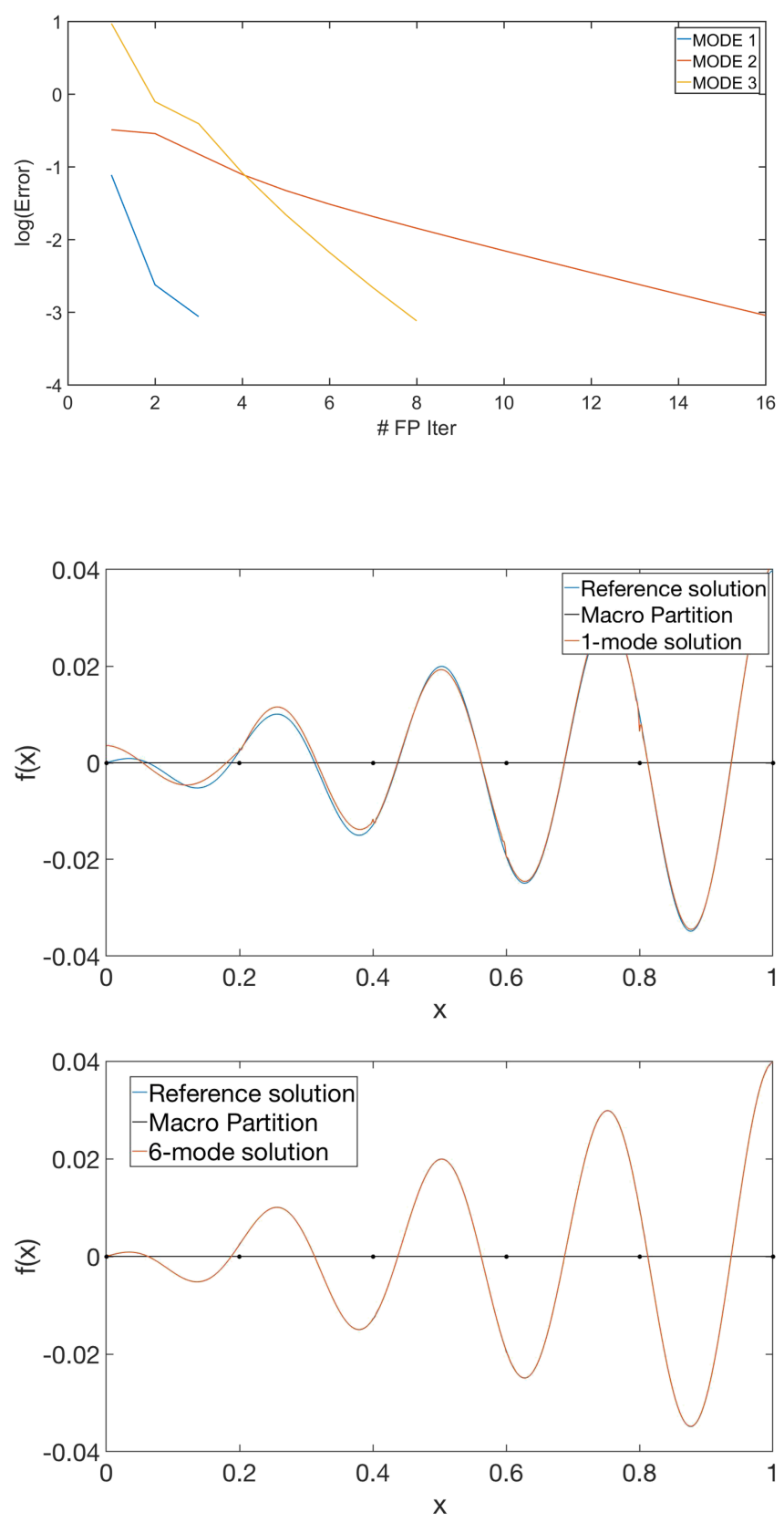

FIGURE 6 Alternate direction scheme convergence for the 1D approximation case with macro partition coincident with a signal period. FP, fixed point

FIGURE 7 Approximation problem. (Top) solution with one single mode. (Bottom) six modes. The reconstructed solution appears as a red line. The reference solution is represented by a blue line. Macro domain partition with six degrees of freedom, appearing as black dots
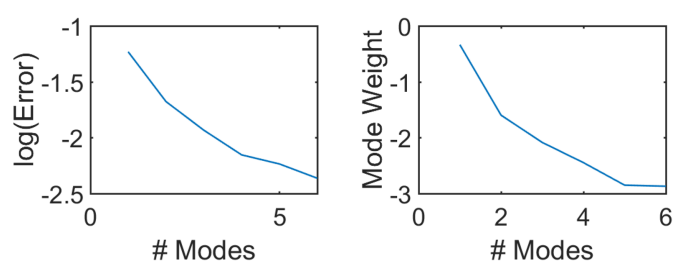

FIGURE 8 One-dimensional approximation problem macro partition noncoincident with a signal period. (Left) relative error with respect to the reference solution as a function of the number of proper generalized decomposition (PGD) modes. (Right) weight of each computed PGD mode [Colour figure can be viewed at wileyonlinelibrary.com]

Figure 9 shows the convergence of the alternated directions algorithm for each one of the modes in the 1D approximation case with macrodomain noncoincident with the signal period. As it can be seen, all modes present a reasonable convergence till the stopping criterion $1 e-3$, with the first mode being the fastest one. The convergence of the fixed point wherever the macromesh is not coincident with the signal period becomes slower compared with the coincident case. This is expected because the functions found by the PGD algorithm are harder to capture in the noncoincident case. 
FIGURE 9 Alternate direction scheme convergence for the 1D approximation case with macro partition noncoincident with a signal period. FP, fixed point

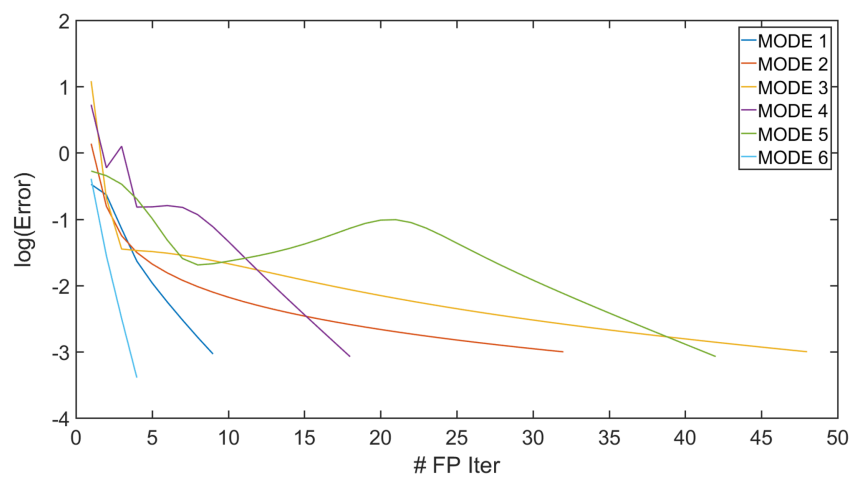

\subsection{2 | Diffusion case}

The second test problem is a pure diffusion equation. The weak form related to this problem is

$$
\int_{\Omega} \frac{D w^{*}(x, \tau)}{D x} \frac{D u(x, \tau)}{D x} d x=\int_{\Omega} w^{*}(x, \tau(x)) f(x) d x, \quad \forall x \in \Omega=[0,10] .
$$

The chain rule is required, as in Equation (5), due to the dependence of $\tau$ with respect to $x$. The source term and the two boundary conditions ensuring that the problem is well posed are

$$
\begin{aligned}
f(x) & =\cos (2 \pi x)+\frac{x}{100}, \\
u(0) & =0 \\
u(10) & =0 .
\end{aligned}
$$

Under these conditions, the analytical solution of the problem reads

$$
u(x)=\frac{\cos (2 \pi x)-1}{4 \pi^{2}}+\frac{x}{6}-\frac{x^{3}}{600}
$$

Figure 10 compares the reconstructed solution using the first PGD mode against the reference solution. The macrodomain has been partitioned using eight dofs. As it can be noticed, the first mode already captures the macro behavior; however, extra modes are required to identify the oscillatory behavior of the microscale. Indeed, the reconstructed solution with three modes already captures the oscillations of the microscale.

Figure 11 shows both the convergence of the reconstructed solution with respect to the number of PGD modes and the weight of each PGD mode. Again, results show good convergence properties toward the reference, exact solution. Note how the weight of subsequent mode decreases monotonically.

Figure 12 shows the convergence of the alternated direction algorithm for each one of the modes in the 1D diffusion case. It can be appreciated how the fixed point converges properly for each one of the modes.

\subsection{3 | Convection case}

The third example is a convection problem, whose weak form reads

$$
\int_{\Omega} w^{*}(x, \tau(x)) \frac{D u(x, \tau(x))}{D x} d x=\int_{\Omega} w^{*}(x, \tau(x)) f(x) d x \quad \forall x \in \Omega=[0,10] .
$$

It is well known that special attention has to be paid when dealing with convection-dominated equations to ensure convergence. A standard SUPG is implemented in this particular case. ${ }^{1}$ Therefore, the test function is defined as

$$
w^{*}(x, \tau(x))=u^{*}(x, \tau(x))+\beta \frac{D u^{*}(x, \tau(x))}{D x},
$$



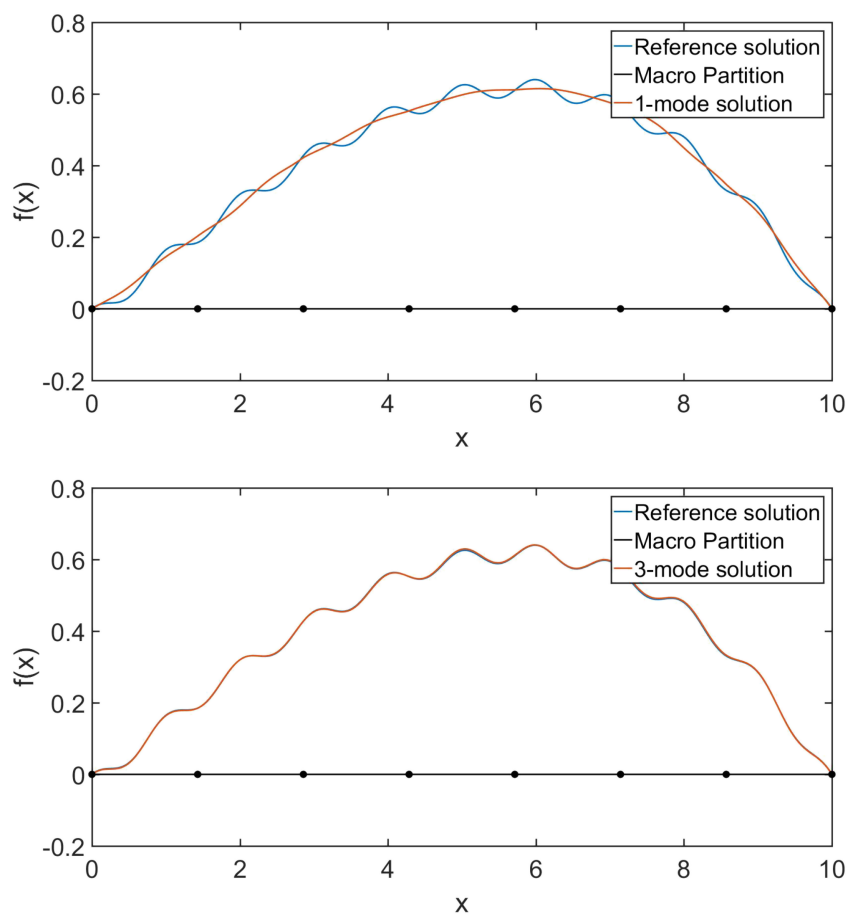

FIGURE 10 Diffusion problem. (Top) one mode. (Bottom) three modes. (Red line) reconstructed solution. (Blue line) reference solution. Macro domain partition with eight degrees of freedom (black dots)
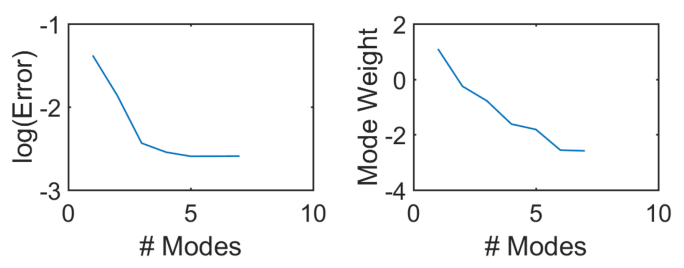

FIGURE 11 One-dimensional diffusion problem. (Left) relative error with respect to the reference solution as a function of the number of proper generalized decomposition (PGD) modes. (Right) weight of each computed PGD mode [Colour figure can be viewed at wileyonlinelibrary.com]

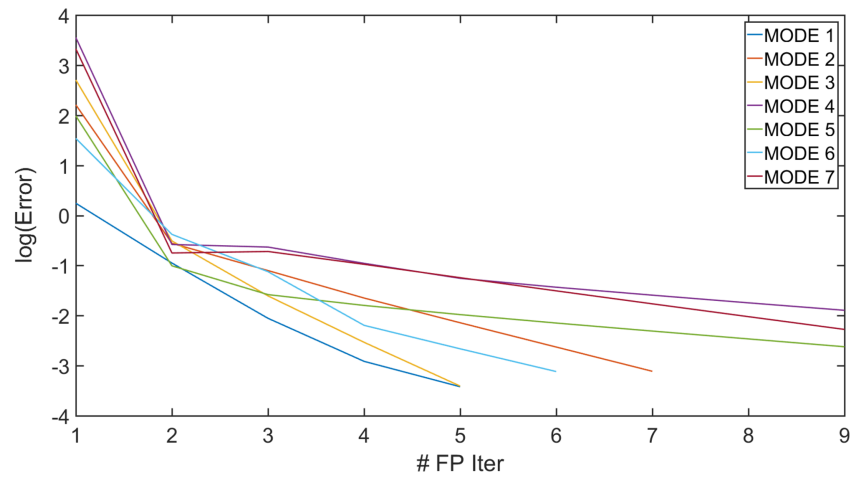

FIGURE 12 Alternate direction scheme convergence for the 1D diffusion case. FP, fixed point

where $\beta$ is a numerical coefficient controlling the stabilization of the numerical scheme. It is important to notice that the numerical scheme remains consistent because the test function affects both sides of Equation (16).

The source term of the problem is

$$
f(x)=x \sin (12 \pi x)+\frac{x}{30},
$$

whereas the initial condition (left boundary) is taken as

$$
u(0)=0 .
$$

In that case, the analytical solution satisfying this initial value problem is

$$
u(x)=\frac{\sin (12 \pi x)}{144 \pi^{2}}-\frac{x \cos (12 \pi x)}{12 \pi}+\frac{x^{2}}{60}
$$


Figure 13 shows the reconstructed solution involving one (top) and seven (bottom) modes versus the reference one. The macrodomain has been partitioned using nine dofs. As it can be noticed, the reconstructed solution is far from the reference one, when only one mode is involved. However, the PGD algorithm converges toward the reference solution when more modes are added into the approximation.

In turn, Figure 14 shows the convergence of the multiscale PGD algorithm for the 1D convection case. The method converges well, reaching a relative error of $10^{-4}$ when using seven modes. It is important to notice that the error seems to stagnate after five PGD modes. Of course, by refining the macroscale, the error decreases.

Figure 15 shows both the convergence of the reconstructed solution with respect to the number of PGD modes and the weight of each PGD mode for the 1D convective case. Again, the methodology shows good convergence properties toward the reference, exact solution. Analyzing more in detail the mode weights, the first four modes are of the same order of magnitude, whereas the rest of them experiences a monotonic decay. The absence of monotonic decay in the mode weights is not surprising because, as proved in classic PGD references, it is only guaranteed when solving symmetric, positive definite operators.

FIGURE 13 Convection case. (Top) one mode. (Bottom) seven modes. The reconstructed solution is represented as a red line, whereas the reference solution appears as a blue line. The macrodomain partition with 10 degrees of freedom is represented as black dots
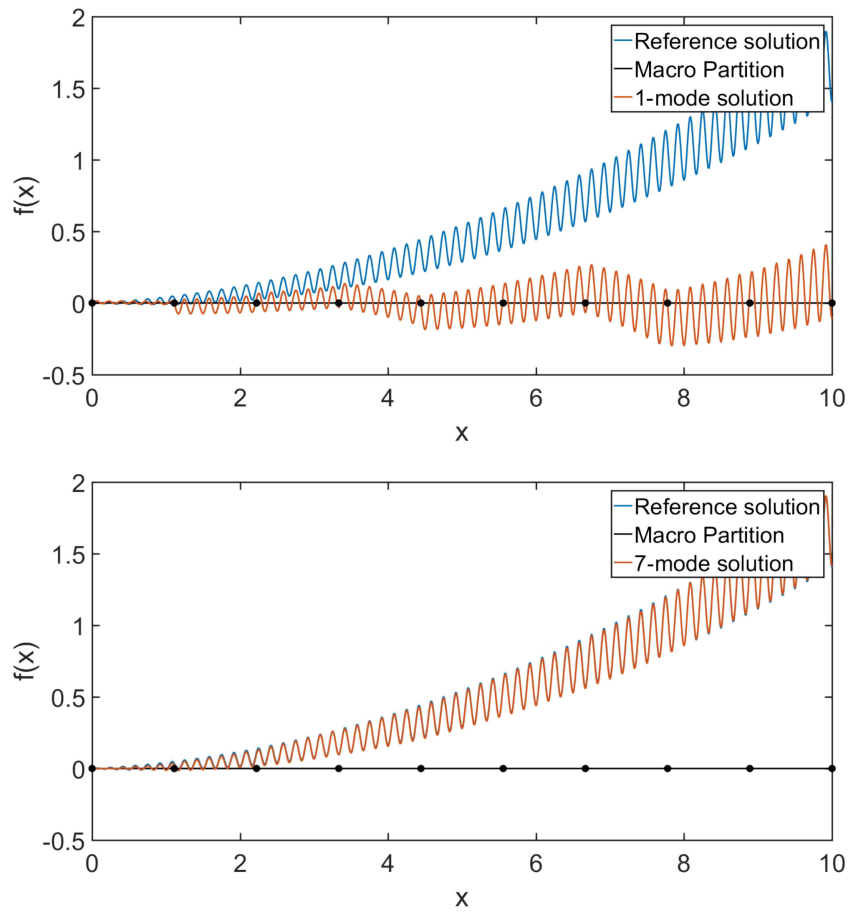

FIGURE 14 Relative error of the reconstructed solution with respect to the reference solution as a number of proper generalized decomposition modes for the convection case [Colour figure can be viewed at wileyonlinelibrary.com]

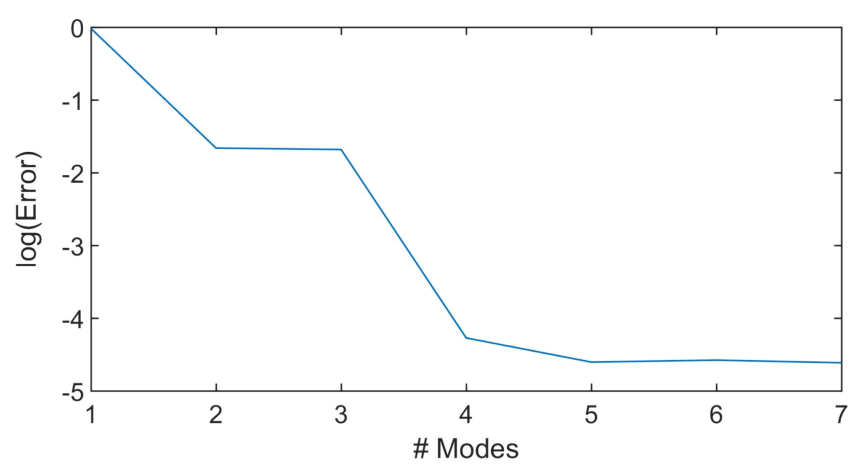

FIGURE 15 One-dimensional diffusion problem. (Left) relative error with respect to the reference solution as a function of the number of proper generalized decomposition (PGD) modes. (Right) weight of each computed PGD mode [Colour figure can be viewed at wileyonlinelibrary.com]
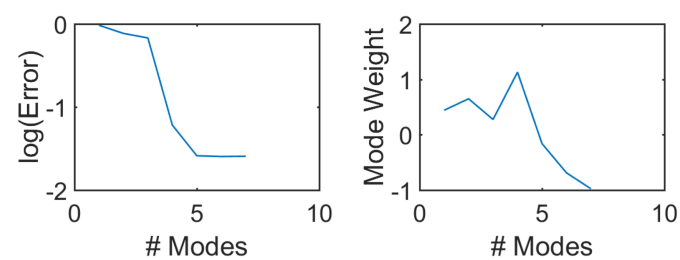


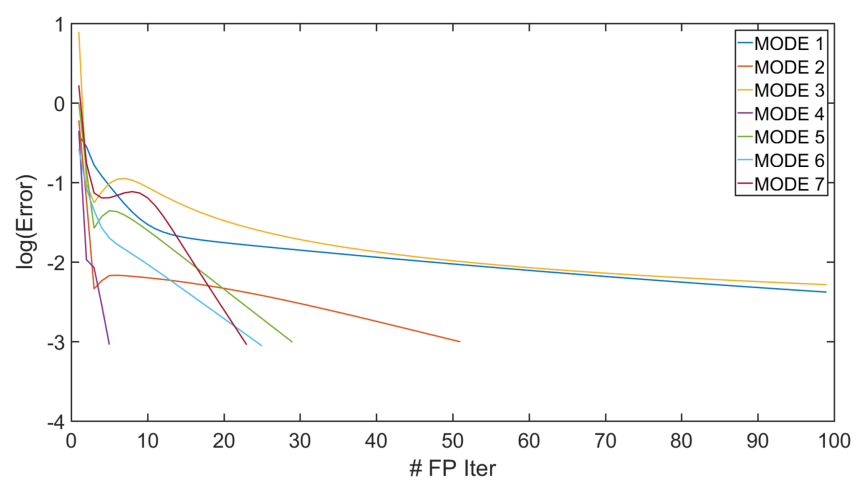

FIGURE 16 Alternate direction scheme convergence for the 1D convection case. FP, fixed point

Figure 16 shows the convergence of the alternated direction algorithm for each one of the modes in the 1D convective case. It can be appreciated how the fixed point converges properly for each one of the modes. However, its convergence may be slow in some particular modes.

\section{2 | Two-dimensional numerical examples}

This section extends the analysis of the developed methodology to three different two-dimensional cases: approximation, diffusion, and convection-reaction-diffusion problems.

\subsection{D approximation problem}

Again, we consider the multiscale approximation of a given function $f(\mathbf{x})$ by a sought function $u(\mathbf{x})$. The weighted residual (Bubnov-Galerkin) form of this problem is

$$
\int_{\Omega} w^{*}\left(\mathbf{x}, \tau_{x}, \tau_{y}\right) u\left(\mathbf{x}, \tau_{x}, \tau_{y}\right) d \mathbf{x}=\int_{\Omega} w^{*}\left(\mathbf{x}, \tau_{x}, \tau_{y}\right) f(\mathbf{x}) d \mathbf{x} \quad \forall \mathbf{x} \in \Omega=[0,1]^{2}
$$

In this case, we consider a function

$$
f(\mathbf{x})=x \cos (8 \pi x) y \cos (8 \pi y) .
$$

Note that no boundary conditions need to be imposed for this problem.

Figure 17 shows the analytical solution (left) versus the reconstructed solution using the multiscale PGD algorithm with three modes. The macrodomain has been partitioned using a mesh of $25 \mathrm{dofs}$, because each spatial direction has been partitioned using five nodes. As it can be clearly seen, the reconstructed solution captures the main features of the reference solution. The small differences are due to the fact that the partition of the macrodomain does not coincide with the period of the signal. Therefore, extra modes will be required to alleviate this nonconformity.

Figure 18 shows the convergence plot associated with the 2D approximation case as a function of the modal enrichments of the solution. Two kinds of indicators are shown: The left plot indicates the L2 relative error of the multiscale PGD solution with respect to the reference solution in logarithmic scale, whereas the right plot shows the L2 norm of each multiscale PGD mode also in logarithmic scale. Note the monotone convergence of the solution just like the monotonic decay in the norm of the modes.

Figure 19 shows the convergence of the alternated direction algorithm for each one of the modes in the approximation case. As it can be seen, all modes present a fast convergence till $1 e-2.5$. Afterwards, all modes are still converging but the convergence slope is decimated.
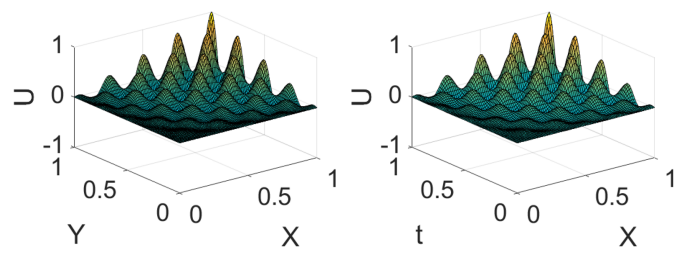

FIGURE 17 Two-dimensional approximation case. (Left) reference solution. (Right) reconstructed solution. The macrodomain is partitioned with $5 \times 5=25$ degrees of freedom [Colour figure can be viewed at wileyonlinelibrary.com] 
FIGURE 18 Two-dimensional approximation case. (Left) L2 relative error of the reconstructed solution with respect to the reference solution. (Right) L2 norm for each multiscale proper generalized decomposition modes [Colour figure can be viewed at wileyonlinelibrary.com]
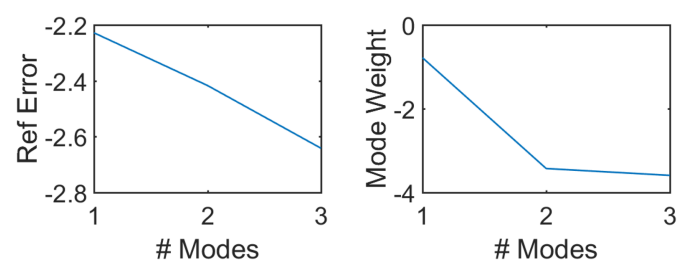

FIGURE 19 Alternate direction scheme convergence for the 2D approximation case. FP, fixed point

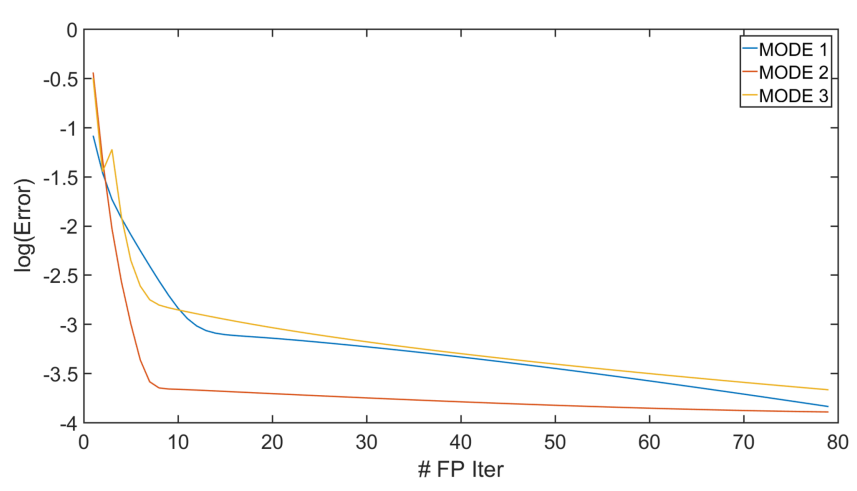

\section{4 | 2D diffusion case}

The weak form of this diffusion problem is

$$
\int_{\Omega} \frac{D w^{*}\left(\mathbf{x}, \tau_{x}, \tau_{y}\right)}{D \mathbf{x}} \cdot \frac{D u\left(\mathbf{x}, \tau_{x}, \tau_{y}\right)}{D \mathbf{x}} d \mathbf{x}=\int_{\Omega} w^{*}\left(\mathbf{x}, \tau_{x}, \tau_{y}\right) f(\mathbf{x}) d \mathbf{x} \quad \forall \mathbf{x} \in \Omega=[0,1]^{2},
$$

with boundary conditions

$$
u(0, y)=u(1, y)=u(x, 0)=u(x, 1)=0 .
$$

The chain rule is required, as shown in Equation (5), because of the dependence of $\tau_{x}$ and $\tau_{y}$ with respect to $x$ and $y$, respectively. The source term $f$ considered in this case was

$$
f(\mathbf{x})=2 w^{2} x y \sin (w x) \sin (w y)-2 w x \sin (w x) \cos (w y)-2 w y \cos (w x) \sin (w y) .
$$

In this particular case, the angular velocity is set to $w=20 \pi$. In that situation, the analytical solution is given by

$$
u(x, y)=x \sin (w x) y \sin (w y)
$$

It is important to highlight the treatment of the boundary conditions to solve the diffusion problem. Vanishing Dirichlet boundary conditions need to be imposed on the boundary, so that all macro dofs placed at the boundary are fixed to zero. To enforce nonvanishing Dirichlet boundary conditions, the first modes will satisfy the Dirichlet boundary conditions, whereas the subsequent modes will be computed with vanishing boundary conditions, following the standard rationale of the PGD. ${ }^{24}$

Figure 20 shows the reference solution for the diffusion case (left) versus the reconstructed solution using five modes of the PGD. The macrodomain has been partitioned using a mesh of four nodes per direction, but because the nodes at the boundary are set to zero, the final dofs are $2 \times 2=4$. Note that there is no perceivable difference between the reference solution and the reconstructed one.

Figure 21 shows both the relative error with respect to the reference solution (left) and the L2 norm of each PGD mode (right) for the 2D diffusion case. The method converges toward the reference solution, and the proposed methodology is also able to extract the multiscale PGD modes ordered in decreasing norm. Therefore, a stopping criterion based on the mode weight seems suitable for this case.

Figure 22 shows the convergence of the alternated direction algorithm for each one of the modes in the diffusion case. All modes are converging until the desired fixed point accuracy. 

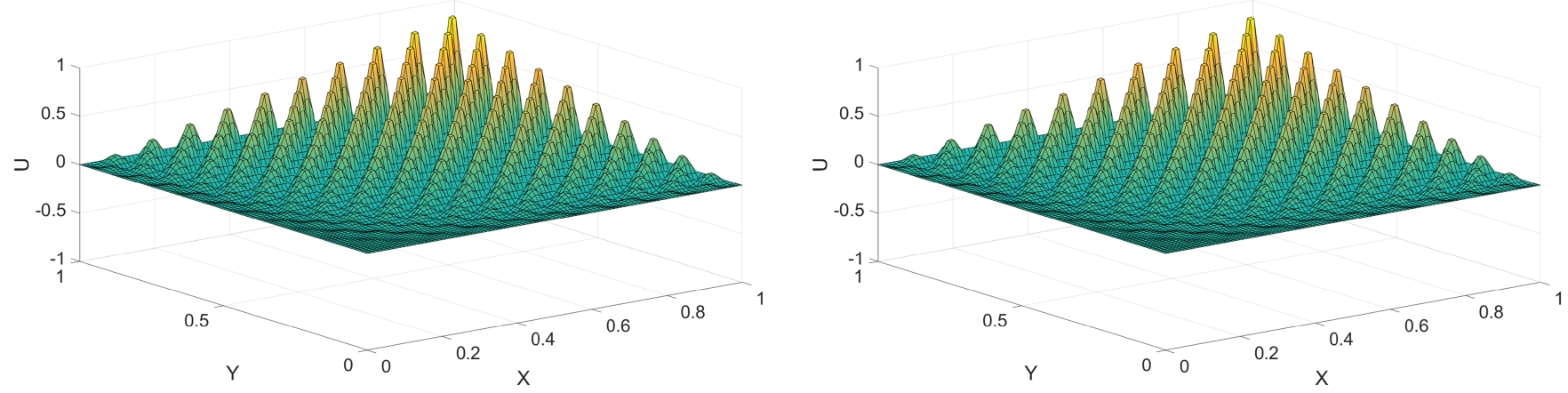

FIGURE 20 Two-dimensional diffusion case. (Left) reference solution. (Right) reconstructed solution with five modes. The macrodomain is partitioned with $2 \times 2=4$ degrees of freedom [Colour figure can be viewed at wileyonlinelibrary.com]
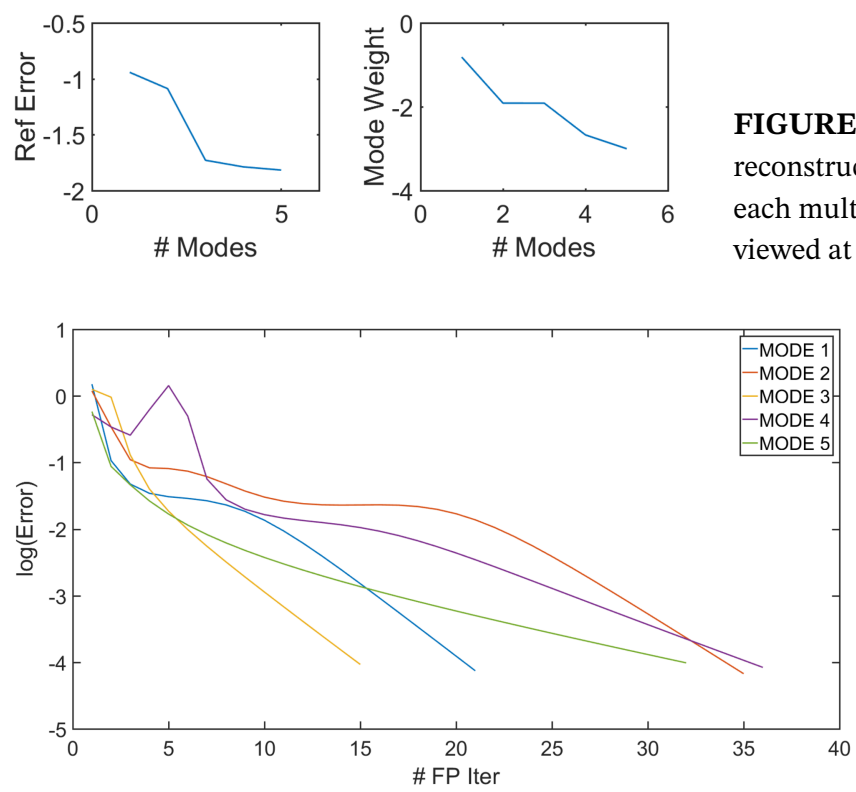

FIGURE 22 Alternate direction scheme convergence for the 2D diffusion case. FP, fixed point

\section{5 | 2D convection-diffusion problem}

The weak form associated with this diffusion problem is

$$
\int_{\Omega} w^{*}\left(\mathbf{x}, \tau_{x}, \tau_{y}\right) \frac{D u\left(\mathbf{x}, \tau_{x}, \tau_{y}\right)}{D y} d \mathbf{x}+\int_{\Omega} \frac{D w^{*}\left(\mathbf{x}, \tau_{x}, \tau_{y}\right)}{D x} \frac{D u\left(\mathbf{x}, \tau_{x}, \tau_{y}\right)}{D x} d \mathbf{x}=\int_{\Omega} w^{*}\left(\mathbf{x}, \tau_{x}, \tau_{y}\right) f(\mathbf{x}) d \mathbf{x}, \quad \forall \mathbf{x} \in \Omega=[0,1]^{2} .
$$

Note that, along the $y$-direction, there is a pure convection phenomenon, whereas the $x$-direction presents pure diffusion. Hence, the nature of the PDE forces to impose boundary conditions at both ends of the $x$ interval, whereas only initial boundary conditions must be imposed in the $y$-direction. The source term reads in this case

$$
f(\mathbf{x})=\left(16 w^{2} y^{2}+2 y\right) \sin (4 w x)+\left(64 w^{2}+w\right) \sin (8 w x) \sin (w y)
$$

whereas the set of boundary conditions ensuring the well-posedness of the problem reads

$$
u(0, y)=u(1, y)=u(x, 0)=0 .
$$

In this case, the angular velocity is set to $w=\pi$.

Under this set of conditions, the partial differential equation (27) admits a unique solution given by

$$
u(\mathbf{x})=\sin (4 w x) y^{2}+\sin (8 w x) \sin (w y)
$$



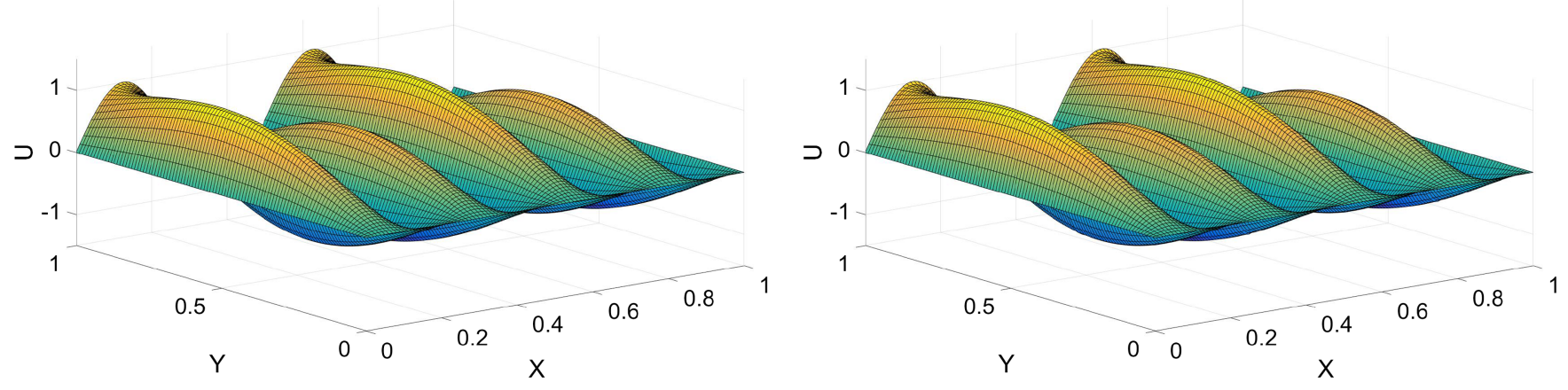

FIGURE 23 Two-dimensional convection-diffusion case. (Left) reference solution. (Right) reconstructed solution with five modes. The macrodomain is partitioned with $3 \times 4=12$ degrees of freedom [Colour figure can be viewed at wileyonlinelibrary.com]

FIGURE 24 Two-dimensional convection-diffusion case. (Left) L2 relative error of the reconstructed solution with respect to the reference solution. (Right) L2 norm for each multiscale proper generalized decomposition modes [Colour figure can be viewed at wileyonlinelibrary.com]
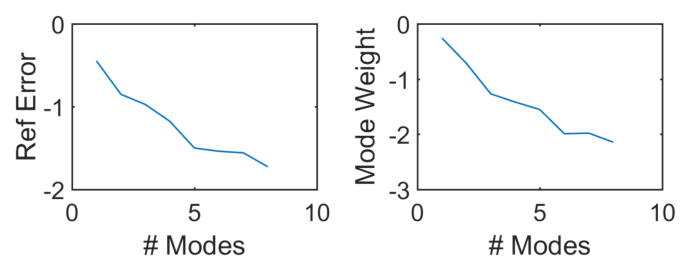

FIGURE 25 Alternate direction scheme convergence for the 2D convection-diffusion case. FP, fixed point

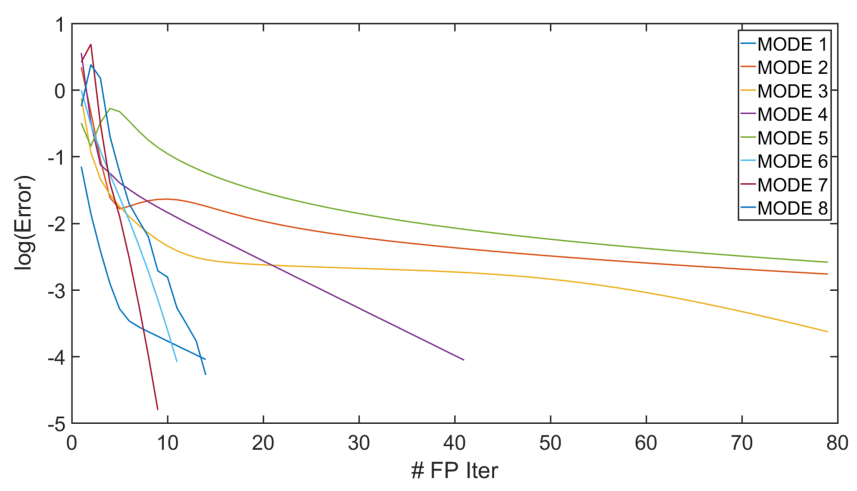

Figure 23 shows the reference solution (left) versus the reconstructed solution involving five modes (right). The macrodomain has been partitioned using $3 \times 4=12$ dofs. As it can be noticed, the reconstructed solution already captures the main features of the reference solution. It is important to notice how the reconstructed solution is able to reproduce the set of homogeneous boundary conditions imposed in the three sides of the squared domain.

The left subplot of Figure 24 shows the relative error versus the number of modes involved in the reconstructed solution for the convection diffusion case. The right subplot depicts the L2 norm of each PGD mode involved in the approximation. The error decreases with respect to the number of modes, showing good convergence properties even for convection diffusion problems. Moreover, the norm of each PGD mode tends to decrease as more PGD modes are involved in the solution.

Figure 25 shows the convergence of the alternated direction algorithm for each one of the modes in the convection diffusion case. It can be appreciated how all modes are converging, yet compared with the pure diffusive case, some modes present slower convergence.

\subsection{Transient thermal problem with multiscale material operator}

The previous $2 \mathrm{D}$ convection diffusion case was the prequel to a more elaborated problem with multiscale material operator. Let us consider a thermal transient problem (time is seen as an extra coordinate) of a 1D bar. If the material properties are homogeneous, the PDE controlling the process is identical to the one studied in the previous convection-diffusion case. However, we are interested in exploring the performance of the methodology when the material parameters also present some variation along the physical space. 

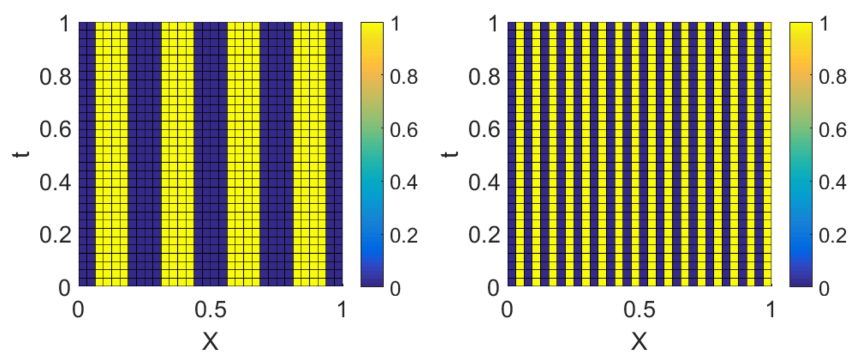

FIGURE 26 Thermal conductivity of the 1D bar in the space-time domain. (Yellow, material A) conductivity equal to 10. (Blue, material B) conductivity equal to 1

Figure 26 shows two test cases where the material parameter presents a variation in the spatial domain. The first one consists of four thick inclusions, whereas the second one is based on 16 thin inclusions.

Since no reference solution is available in this particular case, a reference solution has been computed using full FEM approximation for the entire space-time domain. The mesh used in the 4-inclusion case consists of 6400 linear elements, whereas the one employed in the 16-inclusion case has 9216 elements. The macrodomain is partitioned the same way than in the previous convection diffusion case having 12 total dofs (three dofs in the space domain times four dofs in the time domain). Each microscale unidimensional mode is discretized using 80 piecewise linear elements. The forcing is the same than the one presented in the previous convection-diffusion case as well.

\subsection{1 | Four-inclusion case}

Figure 27 shows the space-time solution for the four-inclusion transient heat problem when using full FEM discretization (left) and multiscale PGD approximation (right). Almost no difference can be appreciated between the full FEM discretization and the multiscale PGD approximation when using eight modes. Note how the derivate of the solution along the space direction is smaller in regions where the material conductivity is higher (yellow material).

Figure 28 shows the convergence of the multiscale PGD toward the full FEM approximation as the number of modes increases (right). Moreover, there exists an important decay of the mode weight as more modes are added to the solution.

Figure 29 shows the convergence of the alternated direction algorithm for each one of the modes for the four-inclusion case. It can be appreciated how all modes are converging, yet some modes require few tens of fixed-point iterations to converge up to the desired tolerance.

In the multiscale context, it is important to use norms other than the standard $L_{2}$ norm to measure the quality of the solution. Indeed, capturing effectively the derivatives of the solution is a major concern for multiscale media. We use an H1-norm defined as

$$
\|u\|_{H 1}=\int_{\Omega} u^{2} d \Omega+\int_{\Omega} \nabla u \cdot \nabla u d \Omega,
$$

whereas the relative error definition is therefore

$$
\mathcal{E}_{H 1}=\frac{\left\|u-u_{r e f}\right\|_{H 1}}{\left\|u_{r e f}\right\|_{H 1}} .
$$
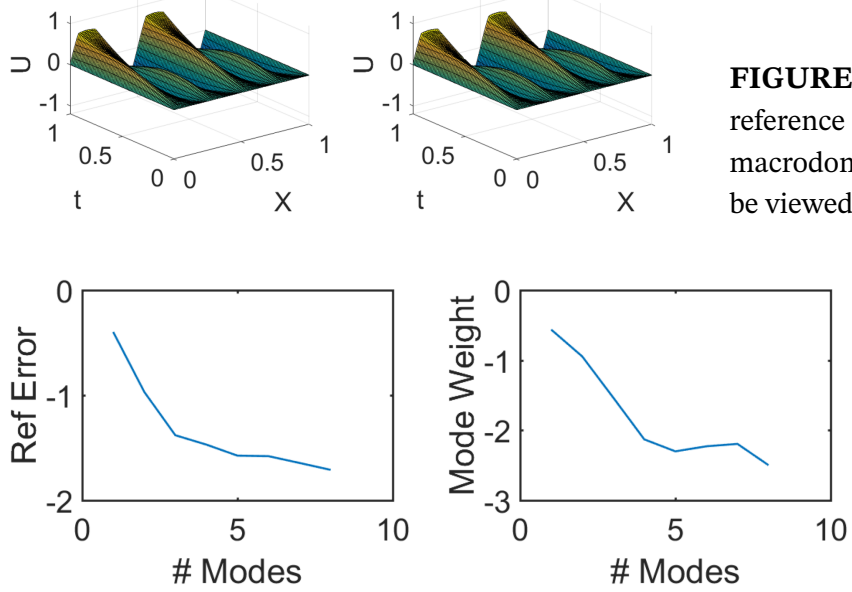

FIGURE 28 Four-inclusion transient thermal case. (Left) L2 relative error of the reconstructed solution with respect to the reference solution. (Right) L2 norm for each multiscale proper generalized decomposition modes [Colour figure can be viewed at wileyonlinelibrary.com] 
FIGURE 29 Alternate direction scheme convergence for the four-inclusion transient heat case. FP, fixed point
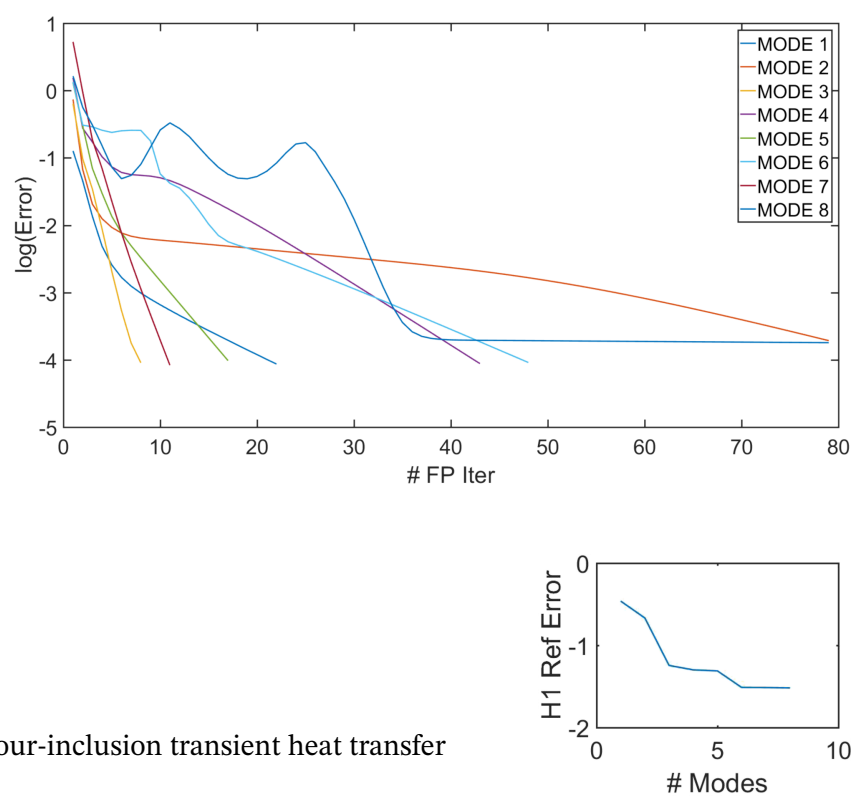

FIGURE $30 \quad H_{1}$-norm error convergence in logarithmic scale for the four-inclusion transient heat transfer case [Colour figure can be viewed at wileyonlinelibrary.com]

Figure 30 shows the $H_{1}$-norm error convergence in logarithmic scale for different PGD modes. As it can be noticed, the error is monotonically decreasing with an increasing number of PGD modes. However, it is important to acknowledge that capturing derivatives within the PGD framework normally requires more enrichments (or modes) than the ones required to capture the primal variable. In other words, a small error in the primal variable may be amplified in the dual variable involving derivatives. Therefore, we would expect that the $H_{1}$-norm presents slower convergence results than the $L_{2}$ indicator for more complicated scenarios, as is the case in standard finite elements.

\subsection{2 | Sixteen-inclusion case}

The same analysis for the 16-inclusion problem already performed for the four-inclusion case is studied in this section. Figure 31 shows the space-time solution for the 16-inclusion transient heat transfer problem when using full FEM discretization (left) and multiscale PGD approximation (right). The effect of the highly varying material parameter can be easily appreciated in the solution. The solution presents plenty of high slopes in the vicinity of the material interfaces. The PGD is in good agreement with the full FEM solution, even though small perturbations are appearing at the interface between macroelements. This fact is arising from the fact that the solution is highly nonlinear at each macropatch, and thus, the multiscale methodology would require more modes to converge as the microscale modes may be very different for each macronodes.

Figure 32 shows the convergence of the multiscale PGD toward the full FEM approximation as the number of modes increases (right) for the 16-inclusion case. The convergence toward the reference solution is slower than the four-inclusion case. This worsening convergence could be easily explained when considering the following two facts. The first one comes from the fact that the same macromesh has been employed for the four-inclusion and 16-inclusion cases. As a consequence, the solution that each individual PGD patch has to replicate is more complicated in the 16-inclusion case compared with the 4-inclusion case, ultimately requiring more modes. The second issue arises from the fact each separated microfunctions is replicated throughout the entire domain, being weighted by the macroscale nodes. That said, another valuable route is to consider a finer macro partition for the 16-inclusion case, being aware that it will require less number of modes but the price to compute one mode will increase.

As mentioned before, because each local microscale path has to replicate a very different nonlinear behavior, it is reasonable to expect that the multiscale PGD methodology will require more modes to converge to the solution.

FIGURE 31 Sixteen-inclusion transient thermal problem case. (Left) full FEM reference solution. (Right) reconstructed solution with eight modes. The macrodomain is partitioned with $3 \times 4=12$ degrees of freedom [Colour figure can be viewed at wileyonlinelibrary.com]
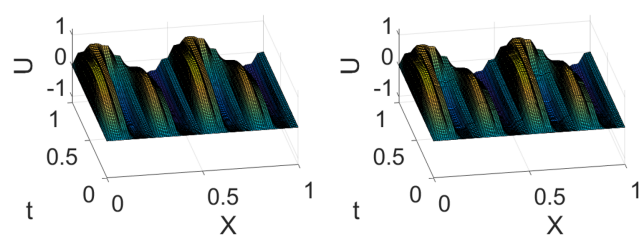

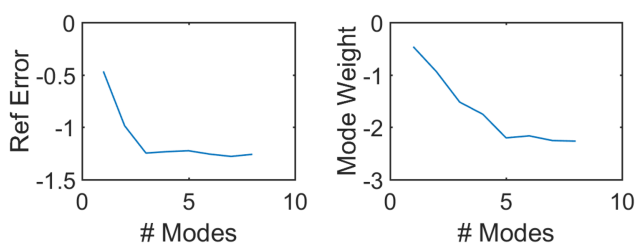

FIGURE 32 Sixteen-inclusion transient thermal case. (Left) L2 relative error of the reconstructed solution with respect to the reference solution. (Right) L2-norm for each multiscale proper generalized decomposition modes [Colour figure can be viewed at wileyonlinelibrary.com]

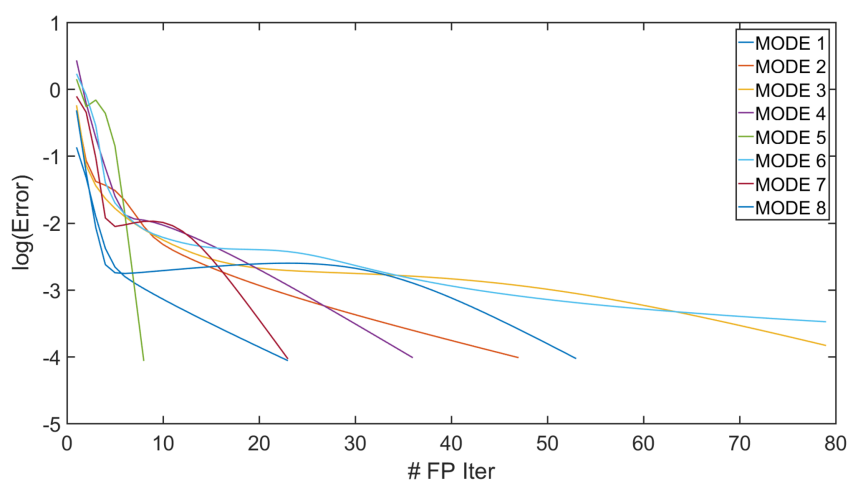

FIGURE 33 Alternate direction scheme convergence for the sixteen-inclusion transient heat case. FP, fixed point

Figure 33 shows the convergence of the alternated direction algorithm for each one of the modes for the 16-inclusion case. It can be appreciated how all modes are converging and some modes require few fixed-point iterations to converge up to the desired tolerance.

\subsection{Time multiscale applied to a discrete system of equations}

The main aim of this example is to show the capabilities of the algorithm to predict the response of a transient system of equations arising from a standard FEM discretization in space. The strong form of the problem reads

$$
\frac{\partial u(x, t)}{\partial t}-\frac{\partial^{2} u(x, t)}{\partial x^{2}}=f(x, t)
$$

Indeed, the problem analyzed in this section makes reference to a $1 \mathrm{D}$ transient thermal problem subjected to the following boundary conditions and source term

$$
\begin{aligned}
& f(x, t)=\left(4 \pi^{2}(\sin (t)+\sin (50 t))+\cos (t)+50 \cos (50 t)\right) \sin (2 \pi x) \\
& u(x, 0)=0 \\
& u(0, t)=u(1, t)=0
\end{aligned}
$$

This partial differential equation under this set of boundary conditions admits an analytical solution

$$
u(x, t)=(\sin (t)+\sin (50 t)) \sin (2 \pi x)
$$

which presents a two-scale behavior in the time domain.

If the spatial 1D domain is discretized using 80 linear elements equally spaced, it gives the discrete system of equations

$$
\mathbf{M} \dot{\mathbf{u}}(t)+\mathbf{K u}(t)=\mathbf{f}(t) \in t=[0, T]
$$

where multiscale is applied only in time.

Figure 34 shows the reference solution (left) and the reconstructed solution (right) using three modes for the discretized transient thermal problem. As it can be noticed, the obtained solution is in good agreement even when involving only one mode. This fact is not surprising because the analytical solution composed by three separated modes involved produces a relative error with respect to the analytical solution of $1 e-2.4$, as shown in Figure 35. 
FIGURE 34 Discrete case. (Left) reference solution. (Right) reconstructed solution with three modes. The temporal domain is partitioned with four macro degrees of freedom [Colour figure can be viewed at wileyonlinelibrary.com]

FIGURE 35 Discretized transient problem. (Left) L2 relative error of the reconstructed solution with respect to the reference solution. (Right) L2 norm for each multiscale proper generalized decomposition modes [Colour figure can be viewed at wileyonlinelibrary.com]
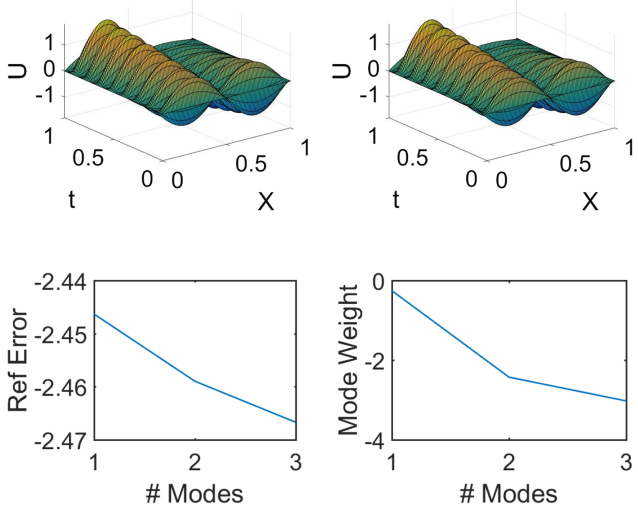

FIGURE 36 Alternate direction scheme convergence for the spatially discretized transient system. FP, fixed point

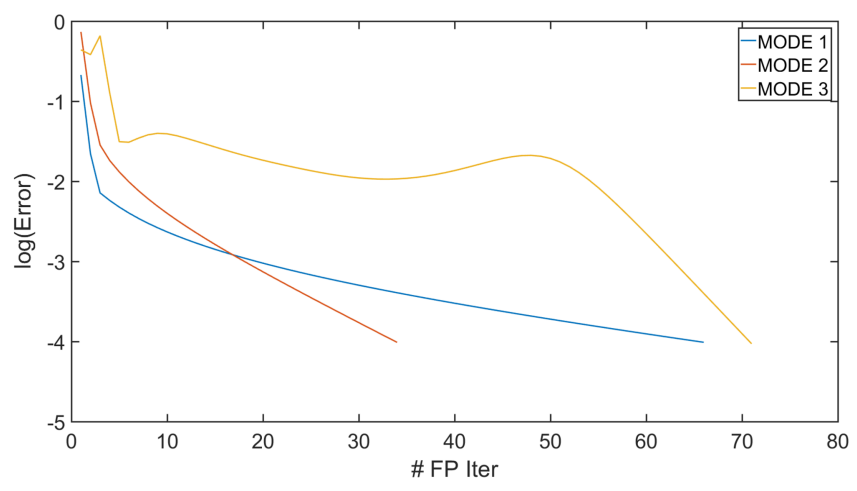

The convergence of the alternate direction scheme for this discretized transient problem is shown in Figure 36 . As it can be seen, the fixed-point procedure reaches the stopping criteria for the three PGD modes composing the solution.

\section{4 | CONCLUSIONS}

A novel algorithm able to solve multiscale problems in an efficient way is proposed in this work. The algorithm combines a macro partition of the domain, which is enriched within the PU rationale. The resulting multiscale multidimensional model is solved using the PGD separated representation. The convergence of the algorithm is proven to be very effective for 1D and 2D problems, involving algebraic, diffusion, and convection phenomena.

The methodology renders good results even when the macrodomain partition does not involve a pure periodical signal in the microscale. The extension of the algorithm to higher-dimensional spaces is our current line of research just like the extension of the methodology to nonrectangular domains.

\section{ACKNOWLEDGEMENTS}

This project has received funding from the European Union's Horizon 2020 research and innovation program under the Marie Sklodowska-Curie grant agreement 675919, by the Spanish Ministry of Economy and Competitiveness through grant DPI2015-72365-EXP, and by the Regional Government of Aragon and the European Social Fund (research group T24 17R).

\section{ORCID}

Elías Cueto (D) https://orcid.org/0000-0003-1017-4381

\section{REFERENCES}

1. Donea J, Huerta A. Finite Element Methods for Flow Problems. Chichester, UK: John Wiley and Sons; 2003.

2. Michel JC, Moulinec H, Suquet P. Effective properties of composite materials with periodic microstructure: a computational approach. Comput Methods Appl Mech Eng. 1999;172:109-143.

3. Feyel F. Multiscale $F E^{2}$ elastoviscoplastic analysis of composite structures. Comput Mater Sci. 1999;16(1-4):344-354. 
4. Hughes T, Feijóo G, Mazzei L, Quincy JB. The variational multiscale method-a paradigm for computational mechanics. Comput Methods Appl Mech Eng. 1998;166:3-24.

5. Codina R, Badia S, Baiges J, Principe J. Variational multiscale methods in computational fluid dynamics. In: Encyclopedia of Computational Mechanics. Hoboken, NJ: John Wiley and Sons; 2017:1-28. https://doi.org/10.1002/9781119176817.ecm2117

6. Passieux JC, Ladevèze $\mathrm{P}$, Néron $\mathrm{D}$. A scalable time-space multiscale domain decomposition method: adaptive time scale separation. Computational Mechanics. 2010;46:621-633. https://doi.org/10.1007/s00466-010-0504-2

7. Fritzen F, Leuschner M. Reduced basis hybrid computational homogenization based on a mixed incremental formulation. Comput Methods Appl Mech Eng. 2013;260:143-154.

8. Fritzen F, Hassani MR. Space-time model order reduction for nonlinear viscoelastic systems subjected to long-term loading. Meccanica. 2018;53:1333-1355. https://doi.org/10.1007/s11012-017-0734-X

9. Maday Y, Turinici G. A parareal in time procedure for the control of partial differential equations. Comptes Rendus Math. 2002;335(4):387-392.

10. Maday Y, Riahi M, Salomon J. Parareal in time intermediate targets methods for optimal control problems. In: Control and Optimization with PDE Constraints. Based on the International Workshop on Control and Optimization of PDEs, Mariatrost, Austria, October 10-14, 2011. Basel, Switzerland: Springer Basel; 2013:79-92.

11. Ammar A, Chinesta F, Cueto E, Doblaré M. Proper generalized decomposition of time-multiscale models. Int J Numer Methods Eng. 2012;90:569-596.

12. Chinesta F, Chaidron G, Poitou A. On the solution of the Fokker-Planck equations in steady recirculating flows involving short fiber suspensions. J Non-Newtonian Fluid Mech. 2003;113:97-125.

13. Chevreuil M, Nouy A, Safatly E. A multiscale method with patch for the solution of stochastic partial differential equations with localized uncertainties. Comput Methods Appl Mech Eng. 2013;255:255-274.

14. Chinesta F, Leygue A, Bordeu F, et al. PGD-based computational vademecum for efficient design, optimization and control. Arch Comput Methods Eng. 2013;20:31-59.

15. Niroomandi S, Alfaro I, González D, Cueto E, Chinesta F. Model order reduction in hyperelasticity: a proper generalized decomposition approach. Int J Numer Methods Eng. 2013;96:129-149.

16. Abdulle A, Bai Y. Reduced basis finite element heterogeneous multiscale method for high-order discretizations of elliptic homogenization problems. J Comput Phys. 2012;231:7014-7036.

17. Boyaval S. Reduced-basis approach for homogenization beyond the periodic setting. Multiscale Model Simul. 2008;7(1):466-494.

18. Efendiev Y, Galvis J, Thomines F. A systematic coarse-scale model reduction technique for parameter-dependent flows in highly heterogeneous media and its applications. Multiscale Model Simul. 2012;10(4):1317-1343.

19. Badías A, González D, Alfaro I, Chinesta F, Cueto E. Local proper generalized decomposition. Int J Numer Methods Eng. 2017;112(12):1715-1732.

20. Babuška I, Melenk JM. The partition of unity method. Int J Numer Methods Eng. 1997;40:727-758.

21. Melenk JM, Babuška I. The partition of unity finite element method: basic theory and applications. Comput Methods Appl Mech Eng. 1996;139(1-4):289-314.

22. Ibáñez R, Abisset-Chavanne E, Chinesta F, Huerta A, Cueto E. A local multiple proper generalized decomposition based on the partition of unity. Int J Numer Methods Eng. 2019. Early view.

23. Chinesta F, Ladevèze P, Cueto E. A short review on model order reduction based on proper generalized decomposition. Arch Comput Methods Eng. 2011;18(4):395.

24. González D, Ammar A, Chinesta F, Cueto E. Recent advances on the use of separated representations. Int J Numer Methods Eng. 2010;81(5):637-659.

25. Chinesta F, Cueto E. PGD-Based Modeling of Materials, Structures and Processes. Cham, Switzerland: Springer International Publishing; 2014.

26. Cueto E, González D, Alfaro I. Proper Generalized Decompositions: An Introduction to Computer Implementation With Matlab. Cham, Switzerland: Springer International Publishing; 2016.

27. González D, Cueto E, Chinesta F, Diez P, Huerta A. SUPG-based stabilization of proper generalized decompositions for high-dimensional advection-diffusion equations. Int J Numer Methods Eng. 2013;94(13):1216-1232.

28. Trottenberg U, Oosterlee CW, Schüller A. Multigrid. London, UK: Academic Press; 2000. 


\section{APPENDIX}

\section{ELEMENTAL OPERATORS}

The main aim of the appendix is to give details about the construction of the operators required to make the multiscale PGD approximation for the different two-dimensional PDEs analyzed in the paper. It is worth to mention that the potential of the PGD algorithm is achieved when all expressions appearing in the formulation are separable. Therefore, we will assume that the macro shape functions appearing in a $2 \mathrm{D}$ problem are separable,

$$
N_{i}(x, y)=N_{i}^{x}(x) N_{i}^{y}(y)
$$

Note that this is the case, for instance, of linear rectangular elements. Nevertheless, the methodology could be applied to more complex elements, involving more terms in the separation of the shape function.

\section{A.1 | Diffusive operator}

In this subsection, a diffusion of a scalar field $u(x, y)$ along the $x$-direction is developed. The derivation for the diffusion along the $y$-direction follows a similar rationale. For the sake of simplicity but without losing generality, we will also assume that a new mode in the macrodirection is desired. Therefore, all variations related to the micro $x$ - and $y$-directions are set to zero.

$$
\begin{aligned}
\int_{\Omega_{e}} \frac{D u^{*}}{D x} \frac{D u}{D x} d x d y= & \sum_{i=1}^{4} \sum_{j=1}^{4} \sum_{k=1}^{M} \int_{\Omega_{e}} u_{i}^{*}\left(\frac{\partial N_{i}}{\partial x} X_{M} Y_{M}+N_{i} \frac{\partial X_{M}}{\partial x} Y_{M}\right)\left(\frac{\partial N_{j}}{\partial x} X_{k} Y_{k}+N_{j} \frac{\partial X_{k}}{\partial x} Y_{k}\right) u_{j} d x d y \\
= & \sum_{i=1}^{4} \sum_{j=1}^{4} \sum_{k=1}^{M} u_{i}^{*} u_{j} \int_{x} X_{M} \frac{\partial N_{i}^{x}}{\partial x} \frac{\partial N_{j}^{x}}{\partial x} X_{k} d x \int_{y} Y_{M} N_{i}^{y} N_{j}^{y} Y_{k} d y \\
& +\sum_{i=1}^{4} \sum_{j=1}^{4} \sum_{k=1}^{M} u_{i}^{*} u_{j} \int_{x} \frac{\partial X_{M}}{\partial x} N_{i}^{x} \frac{\partial N_{j}^{x}}{\partial x} X_{k} d x \int_{y} Y_{M} N_{i}^{y} N_{j}^{y} Y_{k} d y \\
& +\sum_{i=1}^{4} \sum_{j=1}^{4} \sum_{k=1}^{M} u_{i}^{*} u_{j} \int_{x} \frac{\partial X_{M}}{\partial x} N_{i}^{x} N_{j}^{x} \frac{\partial X_{k}}{\partial x} d x \int_{y} Y_{M} N_{i}^{y} N_{j}^{y} Y_{k} d y \\
& +\sum_{i=1}^{4} \sum_{j=1}^{4} \sum_{k=1}^{M} u_{i}^{*} u_{j} \int_{x} X_{M} \frac{\partial N_{i}^{x}}{\partial x} N_{j}^{x} \frac{\partial X_{k}}{\partial x} d x \int_{y} Y_{M} N_{i}^{y} N_{j}^{y} Y_{k} d y
\end{aligned}
$$

It can be highlighted that four different contributions are appearing because of the application of the chain rule. Furthermore, all terms appearing in the last part of Equation (A2) already present a separated format.

\section{A.2 | Convective operator}

In this subsection, a pure convection of a scalar field $u(x, y)$ along the $x$-direction is derived. It is important to notice that this case is a particular case of a general convection given by the velocity field $\mathbf{v}$, where a separated representation of the velocity field would be required. The derivation for the convection along the $y$-direction is given as an exercise for the reader. For the sake of simplicity but without losing generality, we will also assume that a new mode in the macrodirection 
is desired, being the microscale directions known.

$$
\begin{aligned}
\int_{\Omega_{e}} u^{*} \frac{\partial u}{\partial x} d x d y= & \sum_{i=1}^{4} \sum_{j=1}^{4} \sum_{k=1}^{M} \int_{\Omega_{e}} u_{i}^{*} N_{i} X_{M} Y_{M}\left(\frac{\partial N_{j}}{\partial x} X_{k} Y_{k}+N_{j} \frac{\partial X_{k}}{\partial x} Y_{k}\right) u_{j} d x d y \\
= & \sum_{i=1}^{4} \sum_{j=1}^{4} \sum_{k=1}^{M} u_{i}^{*} u_{j} \int_{x} X_{M} N_{i}^{x} \frac{\partial N_{j}^{x}}{\partial x} X_{k} d x \int_{y} Y_{M} N_{i}^{y} N_{j}^{y} Y_{k} d y \\
& +\sum_{i=1}^{4} \sum_{j=1}^{4} \sum_{k=1}^{M} u_{i}^{*} u_{j} \int_{x} X_{M} N_{i}^{x} N_{j}^{x} \frac{\partial X_{k}}{\partial x} d x \int_{y} Y_{M} N_{i}^{y} N_{j}^{y} Y_{k} d y
\end{aligned}
$$

Once again, all integrals appearing in the last part of Equation (A3) are written already in a separated way, improving the efficiency of the algorithm. 